\title{
Enhancement of Convergence Properties of Adaptive CDMA Receiver over Rayleigh Flat Fading Channel
}

\author{
Kh. El-Barbary ${ }^{*}$, M. Samir ${ }^{\dagger}$, and A. Abd El Wahab ${ }^{\dagger}$
}

\begin{abstract}
CDMA system is a promising multiple access technique which extremely increases the channel capacity and supports multiple-access and multimedia transmission. However, the performance of CDMA system degrades in presence of multipath and dense multiple-access environment .Usually an adaptive filter is utilized to compensate the effect of multipath and multiple- access interference and signal fading over the channel.
\end{abstract}

This paper provides a variable step size algorithm to accelerate convergence of the adaptive filter. The optimum performance is achieved by governing the rate of convergence and the amount of steady state excess mean square error (MSE).The performance is optimized in this paper by two main methods mainly: variable step size parameters and gradient adaptively regularization parameter. The first method is implemented through variable step size least mean square algorithm VSSLMS, modified variable step size MVSS algorithm and variable step size affine projection algorithm VS-APA. On the other hand, the second method is implemented using generalized normalized gradient descent algorithm GNGD and generalized square-error-regularized LMS algorithm GSER-LMS.

This paper concerns with comparative study of the five mentioned adaptive algorithms, (VSSLMS - MVSS - VS-APA - GNGD - GSER-LMS), to overcome the multipath and multiple-access problems of CDMA systems. The mentioned algorithms had the advantages of fast convergence and low steady state MSE, these advantages candidate the mention algorithms to improve the bit error rate (BER) performance of the CDMA system, in the presence of multipath and multiple-access environment. The results indicate that the proposed algorithm provides faster convergence compared with the fixed step size and fixed regularization parameters algorithms.

Keywords: Adaptive filters, normalized least mean square, variable step size NLMS, regularization parameter, DS-CDMA.

\section{Introduction}

Code Division Multiple Access using the Direct Sequence Spread Spectrum technique has many advantages like the increased channel capacity and immunity against jamming. In multiuser CDMA, multiple access interference (MAI) is the main source of performance degradation. The MAI is caused by other users in the channel. The adaptive techniques have been successfully used to equalize the channel and thus reduce the MAI in the DSCDMA system. Reduction of MAI is our prime concern which leads to system improvement.

\footnotetext{
* Staff member, Modern Academy, Egypt, khbar2000@yahoo.com .

${ }^{\dagger}$ Egyptian Armed Forces, Egypt.
} 
In order to implement the CDMA receiver with a bank of matched filters, many parameters for all users such as signal delay/timing, signal phase, signal amplitude, signatures, multipath channel profile, and the number of users have to be known. Therefore, the implementation of the bank of matched filters independently from the rest of the receiver is a difficult task except in a very limited number of cases [1].

In order to overcome the problem of CDMA system parameter estimation, adaptive receivers have been proposed. Two types of adaptive detectors are generally used. Firstly, the blind adaptive detector which is used when the spreading code and the channel parameters are known or can be estimated. Secondly, the trained based implementation which is used in case of absence of this information. A known training sequence is transmitted which is used to tune the coefficient of the adaptive filter before the actual data is send. So, that the receiver converges to its steady state, and thereafter it can be made to run in a decision directed mode. Usually the LMS algorithm is used for varying the weights of the coefficient adaptively. The LMS algorithm is simple and easy to implement, but it converges slowly. To overcome this drawback, many variant of LMS algorithm are proposed. It is well known that the final excess mean error (MSE) is directly proportional to the adaptation step size of the LMS while the convergence time increases as the step size decreases. By optimally selecting the step-size, during the adaptation, we can obtain both fast convergence rate and low steady-state mean square error.

For highly correlated inputs the LMS algorithm has a slow convergence which requires long training sequences and therefore low transmission speeds. Another drawback of the LMS is the trade-off between convergence speed and the steady state error since both are controlled by the same parameter. So we introduce a variable step size with different algorithms which has time varying regularization parameter which is gradient adaptively used to improve both the final excess mean square error (MSE) and the convergence speed.

The normalized least mean square (NLMS) adaptive filter is very popular because of its simplicity and robustness. It is well-know that the stability of NLMS is controlled by a step-size parameter $\mu$, which also controls the speed of convergence, tracking ability and steady-state mis-adjustment of the filter. In practice, the NLMS is implemented by dividing the step-size parameter by the squared norm of the input vector plus a small positive constant $\varepsilon$ called the regularization parameter. The inclusion of $\varepsilon$ alleviates the problem when the squared norm getting too close to zero in certain applications [2]. For signals that have great spectrum, the overall effective step-size might vary radically, and the value of $\varepsilon$ may notably affect the convergence and tracking performance of the $\varepsilon$-NLMS adaptive filter.

There are conflicting objectives between fast convergence and low mis-adjustment for fixed regularized NLMS algorithms. In the past two decades, many variable step-size NLMS (VSS-NLMS) algorithms have been proposed to solve this dilemma of the conventional NLMS .For example, by using the power of the instantaneous error to introduce a (VSSLMS) filter [3]. This VSSLMS has a larger step size when the error is large and a smaller step size when the error is small. But the VSSLMS algorithm is sensitive to the accompanying noise, a modified VSSLMS (MVSS) algorithm is presented to alleviate the influence of uncorrelated disturbance [4].The step size update of MVSS is adjusted by utilize an estimate of the autocorrelation of errors at adjacent time samples. The norm of filter coefficient error vector has been used in [5] as a criterion to determine how close the adaptive filter is to optimize the performance, and they are proposed a variable step size affine projection algorithm (VS-APA) to improve the filter performance. 
Another type of VSS algorithms has time - varying regularization parameter $\varepsilon(n)$ that is fixed in the conventional ( $\varepsilon$-NLMS) filters by making the regularization parameter gradientadaptively, the generalized normalized gradient descent (GNGD) algorithm adapts its learning rate according to the dynamics of the input signals, and its performance is bounded from below by the performance of the NLMS [6]. While most VSS-NLMS algorithms need to tune several parameters for better performance with fast convergence, quick tracking and low steady state MSE. Chen et al. presented a tuning-free generalized square-error-regularized NLMS algorithm (GSER-LMS) [7]. The mentioned regularized NLMS algorithm outperform (GSER-LMS) exhibits very good performance with fast convergence, quick tracking, and low steady - state MSE.

This paper provides a fair comparison among these VSS algorithms to get the most algorithms exhibits very good performance with fast convergence, quick tracking and low steady state MSE.

\section{The System Description}

We consider a chip and bit synchronous BPSK-modulated CDMA system which is summarized in Fig.1. By using a bank of adaptive minimum mean squared error (MMSE) filters have ability to perform joint synchronization, channel parameter estimation, and signal detection where the signal is sent over an unknown and improve bit error rate (BER) performance in a multi cellular mobile communication. Firstly, we will consider the channel to be a Rayleigh flat-fading channel, with AWGN. There are $K$ users, and $(2 M+1)$ bits in the data block transmitted by each user. The adaptive MMSE receiver directly processes samples of the received signal at chip (or fraction of a chip) interval, and operates like an adaptive equalizer, suppressing the interference and detect only the desired user, and all other $(K-1)$ users are interferers. The received signal can be written as:

$r(t)=\sum_{k=1}^{K} \sum_{m=-M}^{M} A_{k} b_{k}(m) c_{k}(m) s_{k}(t-m T)+\sigma n(t)$

where $A_{k}$ is the received amplitude of the $k^{\text {th }}$ user's signal, such that $A_{k}^{2}$ is the power of the $k^{\text {th }}$ user; $b_{k}(m) \in\{-1,+1\}$ is the $m^{\text {th }}$ bit transmitted by the $k^{t h}$ user; $c_{k}(m)=\alpha_{k}(m) e^{j ø \mathrm{k}(m)}$ is the channel coefficient for user $k$, bit $m ; s_{k}(t)$ is the deterministic signature waveform of the $k^{t h}$ user, normalized to give unit energy; the discrete time representation of $s_{k}(t)$, designated by $s_{k}$ is of length $N$ chips per bit, where $N$ is the processing gain; $n(t)$ is white Gaussian noise with unit power spectral density; The received signal, $r(t)$ is sampled once per chip to yield $r_{k}(m)$, which is chip and bit aligned to user $k$.

$$
\boldsymbol{r}_{k}(m)=\left[r_{k 1}(m), r_{k 2}(m), \ldots \ldots \ldots \ldots \ldots, r_{k K}(m)\right]^{T}
$$

where $\boldsymbol{r}_{k}(m)$ is the sampled input vector at the adaptive filter such as;

$$
\boldsymbol{r}_{\mathrm{Km}}=\left[r_{k m}(0), r_{k m}(1), \ldots \ldots \ldots \ldots \ldots, r_{k m}(M-1)\right]^{T}
$$

Let $\mathbf{W}_{k}(m)$ denotes the tap weight vector of the adaptive filter with length $N$,

$$
\mathbf{W}_{k}(m)=\left[w_{0}(m), w_{1}(m) \ldots \ldots \ldots \ldots \ldots, w_{N-1}(m)\right]^{T}
$$


The coefficients $\mathbf{W}_{k}$ are obtained adaptively during a training period. After the training period, the coefficients $\mathbf{W}_{k}$ can be kept fixed during data detection. Alternatively, in a decision directed mode. The filter coefficients are obtained by minimizing the $\operatorname{MSE} E\left[\left|\mathrm{e}_{\mathrm{k}}(\mathrm{m})\right|^{2}\right]$, where $e_{k}(m)=d_{k}(m)-\hat{b}_{k}(m)$ is the error between the desired and actual outputs.

There are many adaptive algorithms that are used to obtain the optimal coefficients $\mathbf{W}_{k}(\boldsymbol{m})$.

The modified MMSE criterion is given by:

$$
\mathrm{E}\left[\left|\mathrm{e}_{\mathrm{k}}(\mathrm{m})\right|^{2}\right]=\mathrm{E}\left[\left|d_{k}(m)-\mathbf{W}_{k}^{H}(m) r_{k}(m)\right|^{2}\right]
$$

where $e_{k}(m)$ is the error signal; $d_{k}(m)$ is the data bit (pilot orbit estimate); [ $\left.\mathbf{W}_{k}^{H}(m)\right]$ indicates the Hermitian operation of the tap weight vector for the adaptive filter. The tap weight vector is adapted by LMS algorithm as:

$$
\mathbf{W}(m+1)=\mathbf{W}(m)+\mu e^{*}(m) \boldsymbol{r}_{x}(m)
$$

where [ ]* denotes the complex conjugate; $\mu$ is the step size of the algorithm; $\boldsymbol{r}_{x}$ is the projection of the received signal vector $\boldsymbol{r}_{k}$ on $\mathbf{W}_{k}$.

\section{Variable Step-Size Algorithms}

In this section, several algorithms including VSSLMS [3], MVSS [4], VS-APA [5,8], GNGD $[7,9]$, and the generalized square-error-regularized GSER-LMS [6,7] ] have been summarized.

\subsection{Variable Step-Size LMS (VSSLMS) Algorithm:}

The squared instantaneous a priori estimation of the error is utilized to update the step size

$$
\mu(\mathrm{n}+1)=\alpha \mu(n)+\gamma e^{2}(n)
$$

where $0<\alpha<1, \gamma>0$ and $\mu(n+1)$ is the restricted in some pre-decided [ $\mu_{\text {min }}, \mu_{\max }$ ]. The constant $\mu_{\max }$ is normally selected near the point of instability of the conventional LMS to provide the maximum possible convergence speed. The value of $\mu_{\min }$ is chosen as a compromise between the desired level of steady state mis- adjustment and the required tracking capabilities of the algorithm. The parameter $\gamma$ controls the convergence time as well as the level of mis-adjustment of the algorithm. So, as $\gamma$ decreased the error is large which causes the step size to increase to provide fast convergence as shown in Fig.2. The filter coefficient vector update recursion is given by:

$\mathbf{W}(\mathrm{n}+1)=\mathbf{W}(n)+\mu(n) e(n) \boldsymbol{x}(n)$

\subsection{Modified Variable step-size (MVSS) algorithm:}

We utilized an estimate of the autocorrelation of e $(n)$ at adjacent time samples to control the update of the variable step size as:

$$
\begin{aligned}
& \mu(\mathrm{n}+1)=\alpha \mu(n)+\gamma P^{2}(n) \\
& P(n)=\beta P(n-1)+(1-\beta) e(n) e(n-1)
\end{aligned}
$$

The use of $P(n)$ in the update of $\mu(n)$ serves two objectives. Firstly, the error autocorrelation is generally a good measure of the proximity to the optimum. Secondly, it rejects the effect of the uncorrelated noise sequence on the step-size update. In the early stages of adaptation, the 
error autocorrelation estimate $P^{2}(n)$ is large, resulting in a large $\mu(n)$.As we approach the optimum, the error autocorrelation approaches zero, resulting in a smaller step size, and 0 $<\beta<1$ is an exponential weighting parameter that governs the averaging time constant, the filter coefficient vector update is the same as mentioned in Equation (8) . Moreover, a Fig.3 show, when $\gamma$ decreased the error is large which causes the step size to increase to provide fast convergence.

\subsection{Variable Step-Size Affine Projection (VSS-APA) Algorithm:}

A variable step- size affine projection algorithm (VSS-APA) employs an error vector, instead of a scalar error as used in VSSLMS, to adjust the variable step size. The coefficient vector recursion is given by:

$\mathbf{W}(\mathrm{n}+1)=\mathbf{W}(n)+\mu(n) \mathbf{x}(n)\left(\mathbf{x}^{\mathbf{T}}(n) \mathbf{x}(n)+\delta_{1} I\right)^{-1} e(n)$

where $\delta_{1}$ a small positive number, $I$ is a unit matrix of size $\mathrm{MxM}$ and $\mathbf{x}(\mathrm{n})$ is an input matrix defined as:

$$
\begin{aligned}
& \mathbf{x}(\mathrm{n})=[\mathrm{x}(n), \mathrm{x}(n-1), \ldots \ldots \ldots \ldots \ldots, \mathrm{x}(n-k+1)]^{T} \\
& \boldsymbol{e}(\mathrm{n})=[e(n), e(n-1), \ldots \ldots \ldots \ldots \ldots, e(n-k+1)]^{T}
\end{aligned}
$$

The variable step size $\mu(n)$ is obtained by;

$$
\mu(\mathrm{n})=\mu_{\max } \frac{\|\mathrm{P}(\mathrm{n})\|^{2}}{\|\mathrm{P}(\mathrm{n})\|^{2}+\delta_{2}}
$$

where $\delta_{2}$ is a positive number proportional to $M, \mu_{\max }<2[10]$, and $\mathbf{P}(n)$ is an $\mathrm{Mx} 1$ vector recursively given by:

$$
\mathbf{P}(n)=\beta \mathbf{P}(n-1)+(1-\beta) \mathbf{x}(n)\left(\mathbf{x}^{\mathbf{T}}(n) \mathbf{x}(n)+\delta_{1} I\right)^{-1} \boldsymbol{e}(n)
$$

As shown in Fig.4, the variation of the rate of convergence of the VS-APA algorithms at various value of the constant $\delta_{2}$ effect on the value of the variable step-size. So, as $\delta_{2}$ increase the adaptive filter performed well with fast convergence and low MSE.

\subsection{Generalized Normalized Gradient Descent (GNGD) Algorithm:}

The GNGD algorithm uses a time- varying regularization parameter $\varepsilon(n)$ calculated as [11]:

$$
\varepsilon(n)=\varepsilon(n-1)-\rho \mu \frac{e(n) e(n-1) \mathbf{x}^{\mathbf{T}}(n) \mathbf{x}(n-1)}{\| \mathbf{x}\left(n-1\left\|^{2}+\right\| \varepsilon\left(n-1 \|^{2}\right.\right.}
$$

where $\rho$ an adaptation parameter needs tuning, and the initial value $\varepsilon(0)$ has to be set as well.

The filter coefficient vector is updated as:

$$
\mathbf{W}(n+1)=\mathbf{W}(n)+\frac{\mu_{c}}{\|\mathbf{x}(n)\|^{2}+\varepsilon(n)}
$$


where $\mu_{c}$ is a fixed step size, The GNGD [6] solves the problem of poor performance and ultimately divergence of NLMS, when the resulting $\frac{\mu}{\|x(n)\|^{2}}$ grows outside the stability bounds $0<\mu<2$. By performing a gradient update of the regularization parameter $\varepsilon$. The GNGD has been shown to converge extremely fast, even in environments where NLMS diverges. The GNGD has the desirable property that it is almost guaranteed not to diverge.

The variation of the rate of convergence of the GNGD algorithm is robust to perturbations of the regularization term $\varepsilon$ and the initialization of the step size adaptation parameter $\rho$. As shown in Fig.5. Also, as $\varepsilon$ increased the GNGD converges extremely fast.

\subsection{Generalized Square-Error-Regularized NLMS (GSER-LMS) Algorithm:}

For the conventional $\varepsilon$-NLMS algorithm, the role of $\varepsilon$ is to prevent the associated denominator from getting too close to zero, so as to keep the filter from divergence. However, in applications of speech signals, a too small $\varepsilon$ may make the denominator very close to zero while a too big $\varepsilon$ will slow down the adaptation of the filter. Lee et al. propose a generalized square-error-regularized LMS which employs the inverse of the weighted square-error as the time-varying regularization parameter [6]. The proposed GSER-LMS algorithm updates $\mathbf{w}(n)$ as follows:

$\mathbf{W}(n+1)=\mathbf{W}(n)+\frac{\mu}{\|\mathbf{x}(n)\|^{2}+\alpha \delta(n)^{-1}} e(n) \mathbf{x}(n)$

where $\alpha$ is a positive parameter that makes the filter more general and the error signal power $\delta(n)$ can be estimated as:

$\delta(n)=(1-\lambda) \delta(n-1)+\lambda e^{2}(n)$

The positive constant $\lambda \leq 1$. The idea behind GSER-LMS is that when $\alpha$ decreased and the error signal power $\delta(n)$ gets bigger, the regularization parameter $\alpha \delta(n)^{-1}$ gets smaller, and the effective step-size becomes relatively large. Consequently, the filter can make bigger adaptation at this mode. When the estimation error is small, $\alpha \delta^{-1}(n)$ gets larger, and it gives smaller effective step-size. At this stage, the adaptive filter makes small adjustment. A good property of GSER-LMS is that, in practice, $\delta(n)$ nor $\delta(n)^{-1}$ gets too close to zero; we observed that the parameter $\alpha$ may affect the performance of GSER-LMS as shown in the simulation in Fig.6.

\section{Simulation Results}

In this section, the performance of the synchronous adaptive CDMA system with various adaptive algorithms VSSLMS, MVSS, VS-APA, GNGD and GSER-LMS is studied. The convergence speeds of these algorithms are compared. The spreading sequences of the users are generated randomly using gold code with a spreading gain $=31$, all the users are assumed to transmit equal power via the channel. The channel is assumed to be Rayleigh flat fading and Additive Gaussian Noise with zero mean and variance=1.All the simulations are performed under identical conditions to make the comparison fair. The simulation are performed using $10^{-5}$ random transmitted bits for each user. The adaptive filter with 31 coefficients, the convergence of the various adaptive algorithms is evaluated 1000 iterations at $\mathrm{SNR}=25 \mathrm{~dB}$. We have used the excess mean square error (MSE) to evaluate the performance of the algorithms in the presence of 6 interfering users (5 MAI) over Rayleigh flat fading. The MSE curves are ensemble over 100 independent runs .All the algorithms had 
fast convergence speed compared with the FSS-LMS algorithm as shown in Fig.7. The performance of all the proposed various adaptive CDMA receivers approaches the performance of the MF in presence of MAI and AWGN channel with fast converge speed and low MSE and has good performance compared with the matched filter over Rayleigh flat fading as shown in Fig.8. We show some simulation results with the following parameters

$$
\begin{gathered}
\text { setup : } \alpha=\beta=0.97, \quad \gamma=5 \times 10^{-5}, \quad \delta_{1}=0.01, \quad \delta_{2}=0.01, \quad \mu_{\max }=1, \quad \mu_{\min }=10^{-3}, \\
\mu_{c}=1, \quad \rho=0.15, \quad \lambda=0.97 .
\end{gathered}
$$

\section{Conclusions}

Many variable step-size NLMS algorithms have been proposed to achieve fast converge rate, rapid tracking, and low misalignment .This paper summarized several promising algorithms to improve the bit error rate (BER) performance of the CDMA system, in the presence of multipath and multiple- access environment and presented a performance comparison by means of extensive simulation. According to the simulation, The VS-APA and the GNGD and GSER-LMS have a significant convergence rate with best performance improvement compared with those algorithms.

\section{References}

[1] Predgrag B.Rapajic, Senior Member, IEEE, and Deva K.Borah, "Adaptive MMSE Maximum Likelihood CDMA Multiuser Detection" IEEE JOURNAL ON SELECTED AREAS IN COMMUNICATIONS, VOL. 17. NO.12, DECEMBER 1999.

[2] Alexander D.Pouarikas, Zayed M. Ramadan "Adaptive filtering primer with Matlab" Library of congress cataloging-in-publication Data, ch8, pp.137-167, 2006.

[3] R. H. Kwong and E. W. Johnston, "A variable step size LMS algorithm," IEEE Transactions on Signal Processing, Vol. 40, pp.1633 - 1642, July 1992.

[4] T. Aboulnasr and K Mayyas, "A robust variable step-size LMS-type algorithm: analysis and simulations," IEEE Transactions on Signal Processing, Vol. 45, No. 3, pp. 631 639, March 1997.

[5] H. C. Shin, A. H. Sayed, and W. J. Song, "Variable step-size NLMS and affine projection algorithms," IEEE Signal Processing Letters, Vol. 11, No. 2, pp. 132 - 135, Feb. 2004.

[6] J. Lee, H. C. Huang, and Y. N. Yang, "The generalized square-error-regularized LMS algorithm," Proceedings of WCECS2008, pp. 157 - 160, Oct. 2008.

[7] Junghsi Lee, Jia-Wei Chen, and Hsu-Chang Huang, "Performance Comparison of Variable Step-Size NLMS Algorithms" Proceedings of the World Congress on Engineering and Computer Science 2009 Vol I,WCECS 2009, October 20-22, 2009, San Francisco, USA.

[8] Y. S. Choi, H. C. Shin, and W. J. Song, "Adaptive regularization matrix for affine projection algorithm," IEEE Transactions on Circuits and Systems II, Express Briefs, Vol. 54, No. 12, pp. 1087-1091, Dec.2007.

[9] D. P. Mandic; "A generalized normalized gradient descent algorithm," IEEE Signal Processing Letters, Vol. 11, No. 2, pp. 115-118, Feb.2004.

[10] Mohammad Shams Esfand Abadi, Vahid Mehrdad, Aziz ollah Gholi pour and Majid Noroozi "A Family of Variable Step-Size Affine Projection Adaptive Filtering Algorithms" International Journal of Computer and Electrical Engineering, Vol. 2, No. 3, June, 2010.

[11] D. P. Mandicet al., "Collaborative adaptive learning using hybrid filters," Proceedings of 2007 IEEE ICASSP, pp. III 921-924, April2007. 


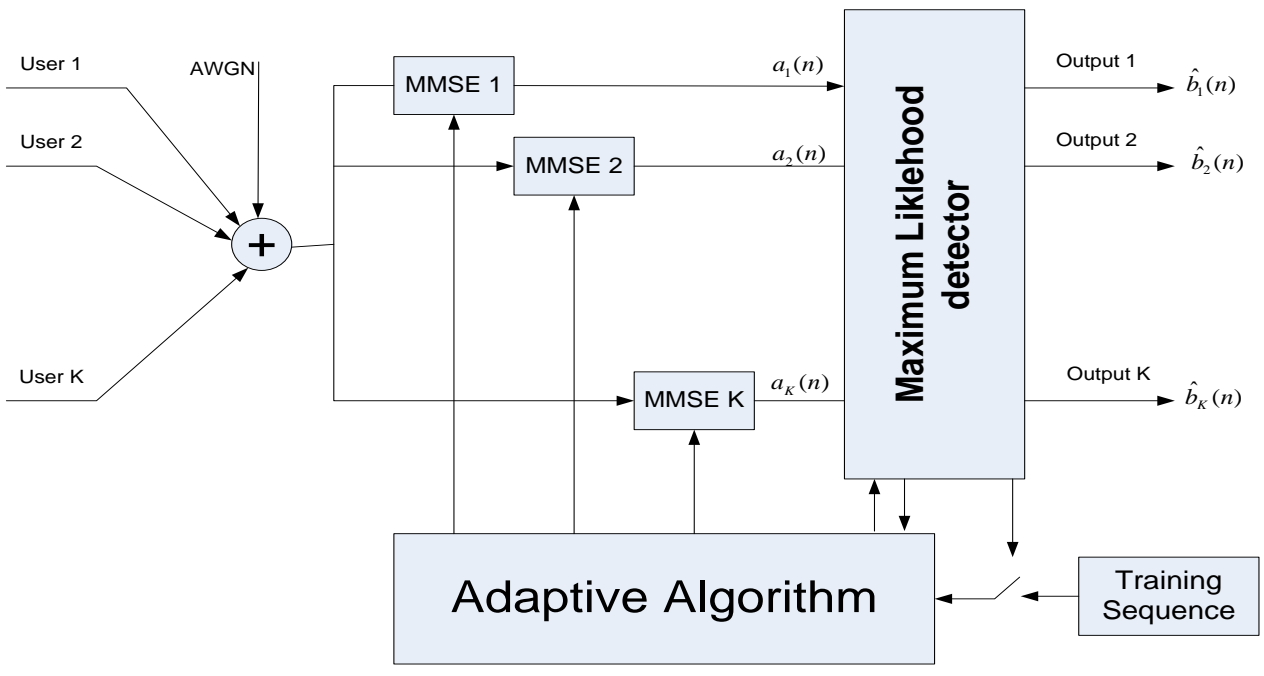

Fig. 1 Simplified Representation of Adaptive CDMA System

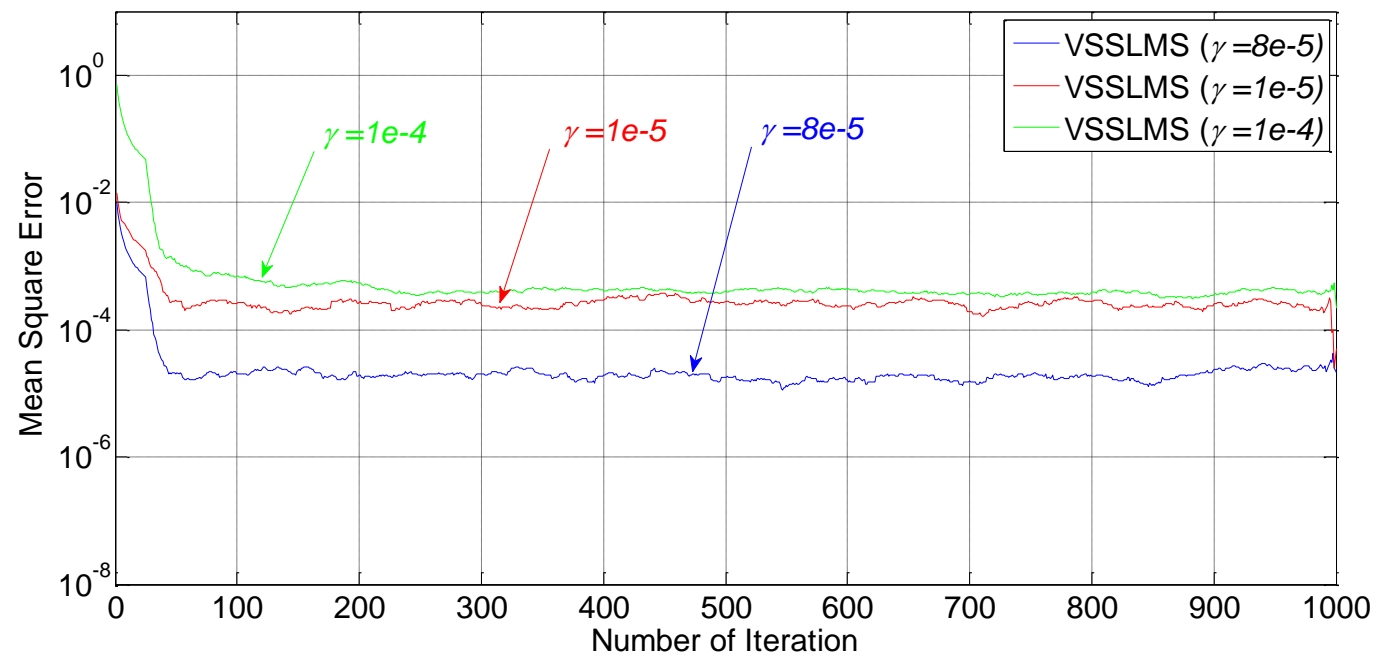

Fig. 2 Variation of the Rate of Convergence of the Proposed VSSLMS as a Function of $(\gamma)$ at $\mathrm{SNR}=25 \mathrm{~dB}$

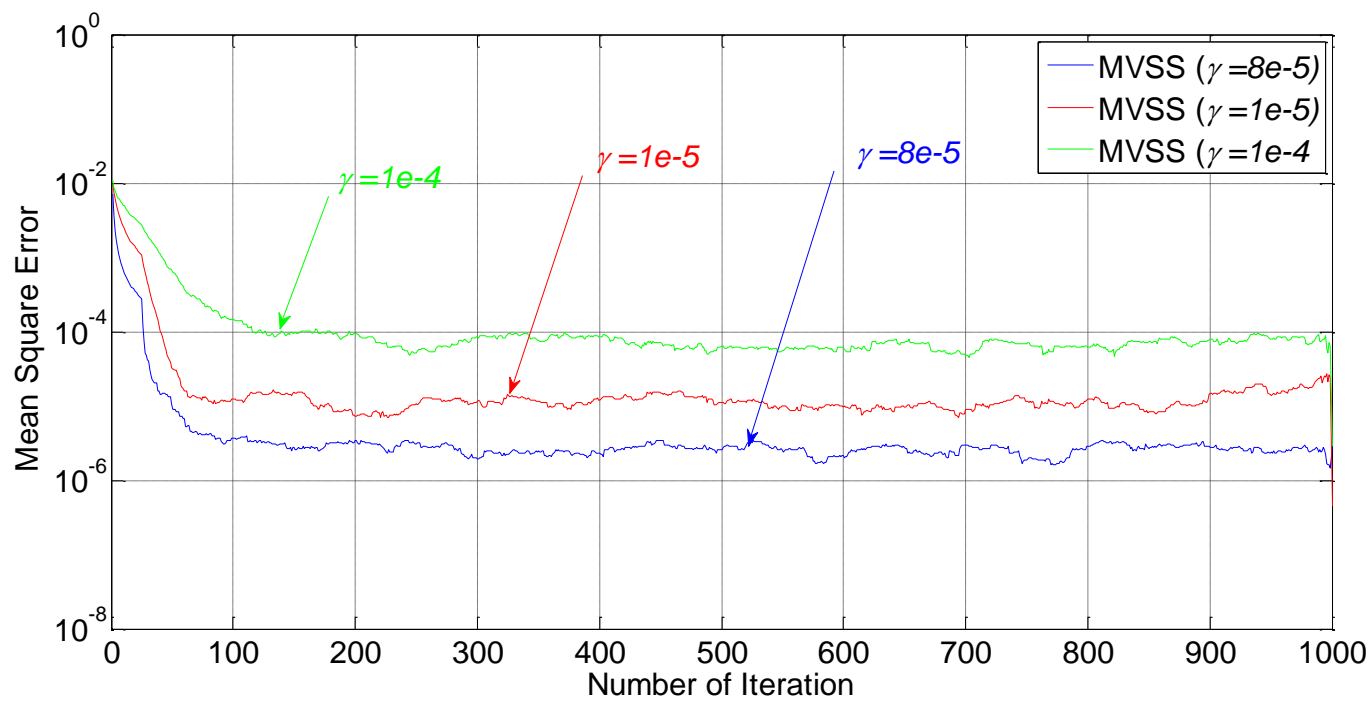

Fig. 3 Variation of the Rate of Convergence of the Proposed MVSS as a Function of $(\gamma)$ at $\mathrm{SNR}=25 \mathrm{~dB}$ 


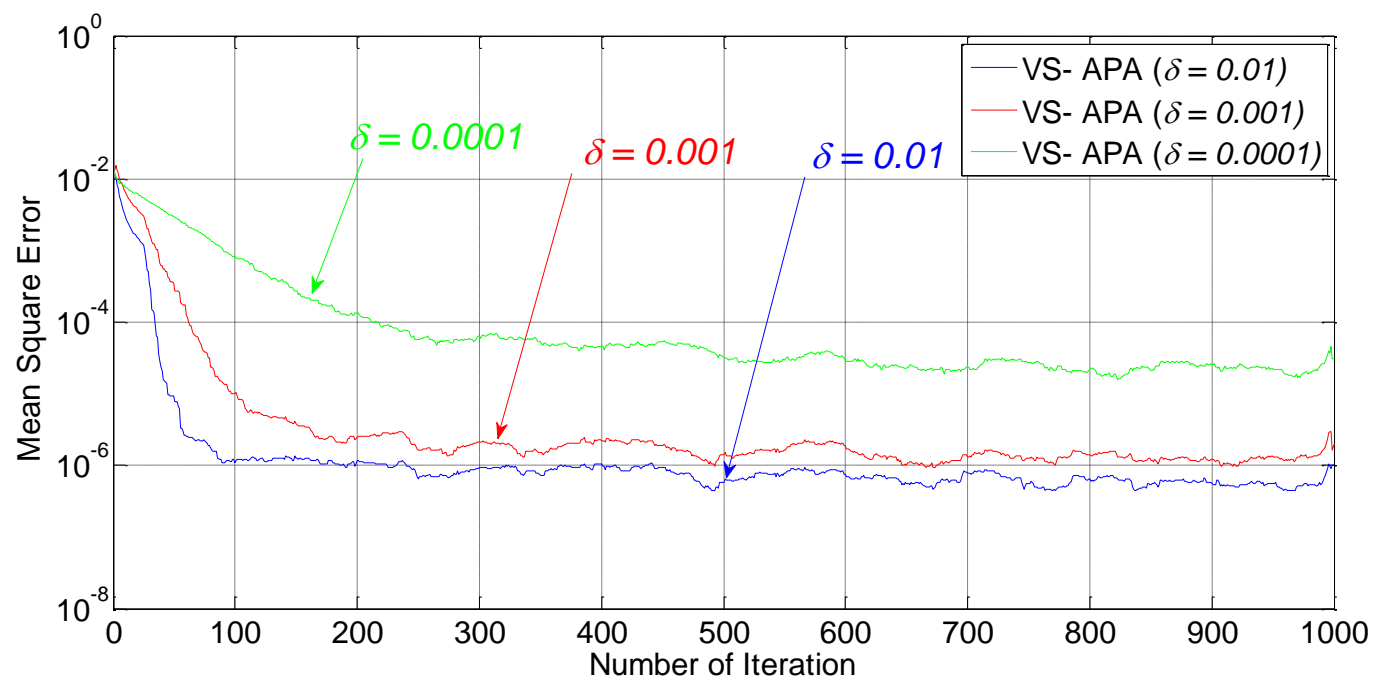

Fig. 4 Variation of the Rate of Convergence of the Proposed VS-APA as a Function of $(\delta)$ at $\mathrm{SNR}=25 \mathrm{~dB}$

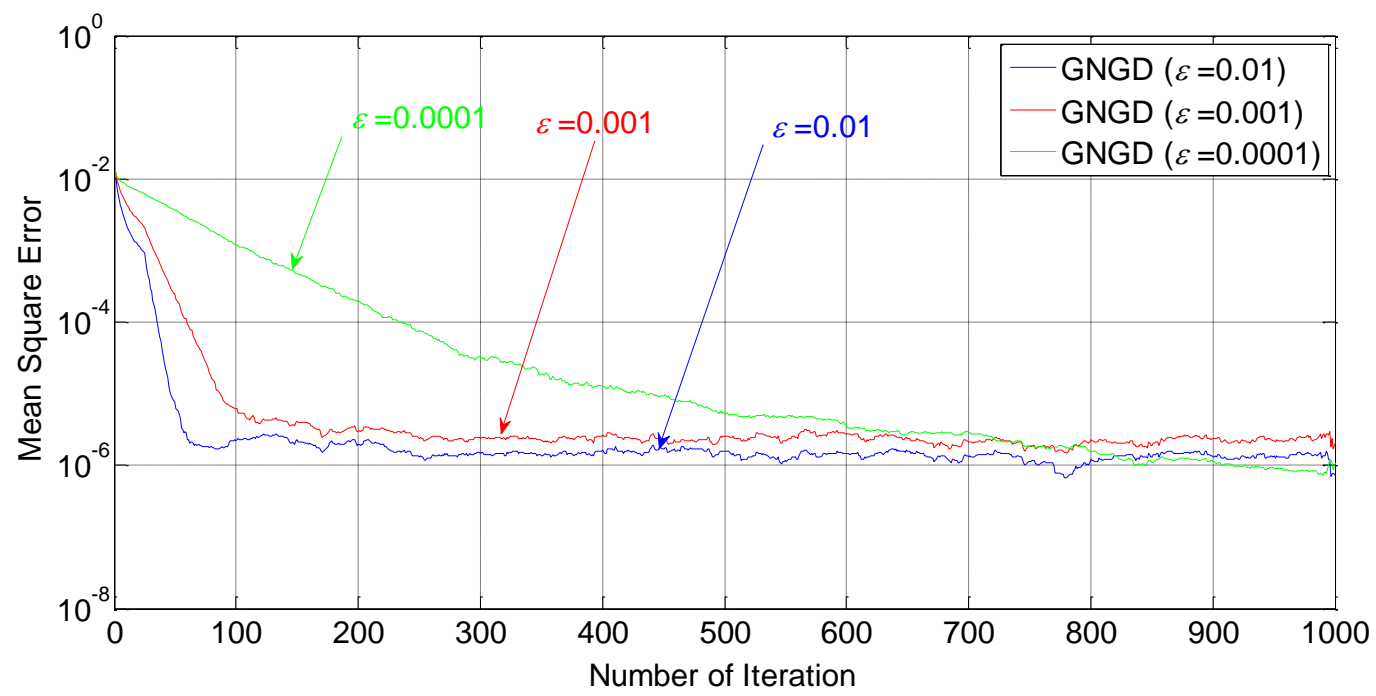

Fig. 5 Variation of the Rate of Convergence of the Proposed GNGD as a Function of $(\varepsilon)$ at $\mathrm{SNR}=25 \mathrm{~dB}$

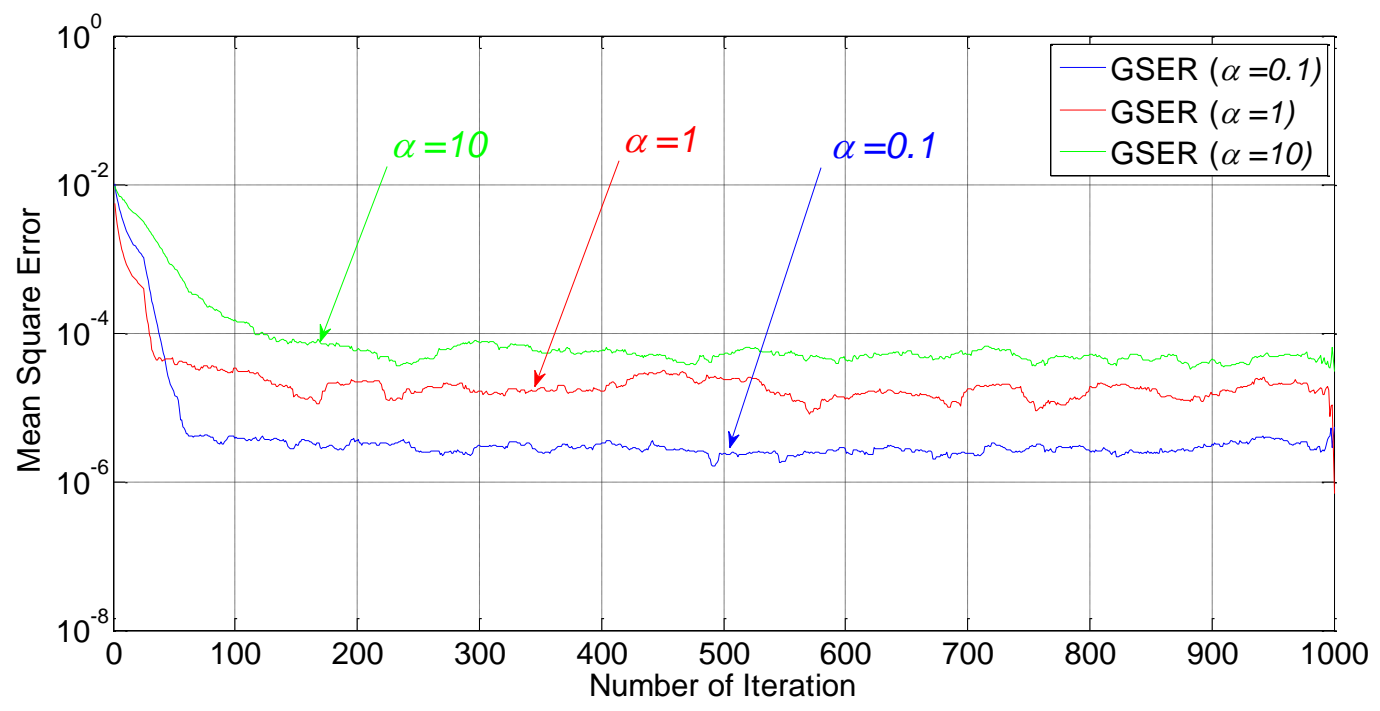

Fig. 6 Variation of the Rate of Convergence of the Proposed GSER-LMS as a Function of $(\alpha)$ at $\mathrm{SNR}=\mathbf{2 5 d B}$ 


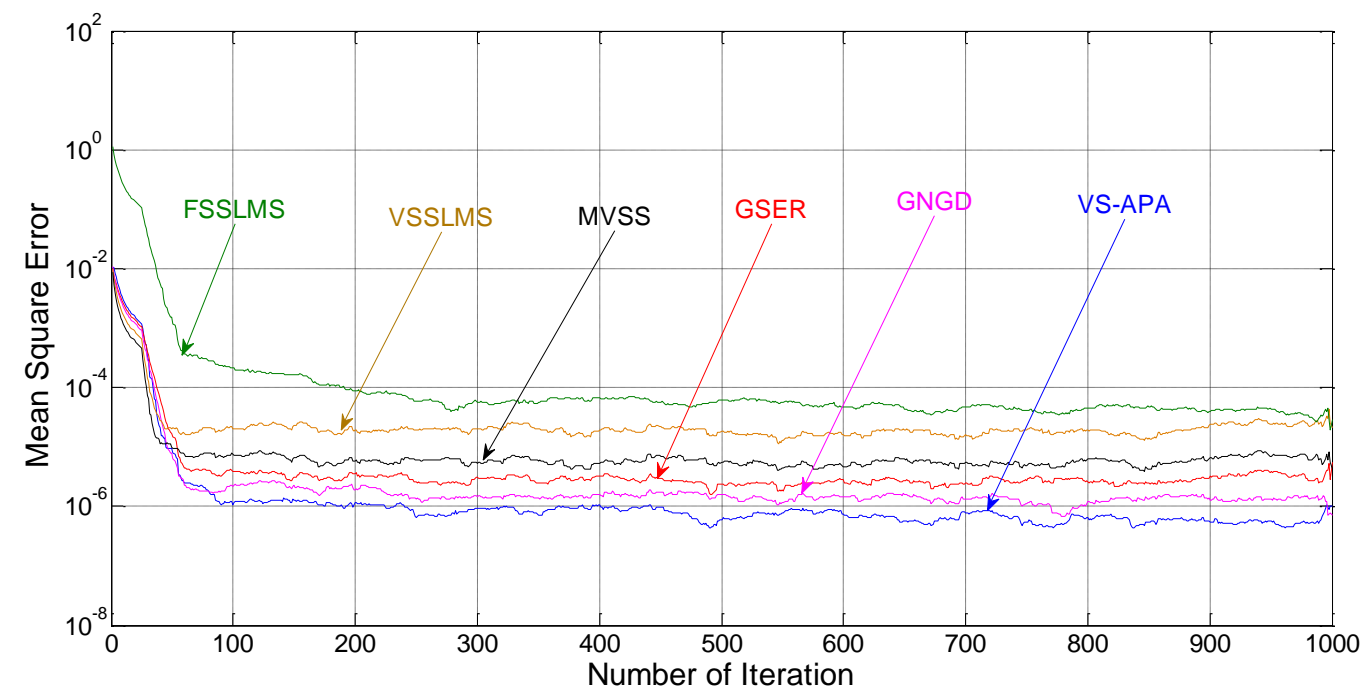

Fig. 7 Convergence of Excess MSE of Various Adaptive Algorithms in CDMA Receivers (Flat Fading, 5 MAI, Gold31) and SNR=25dB

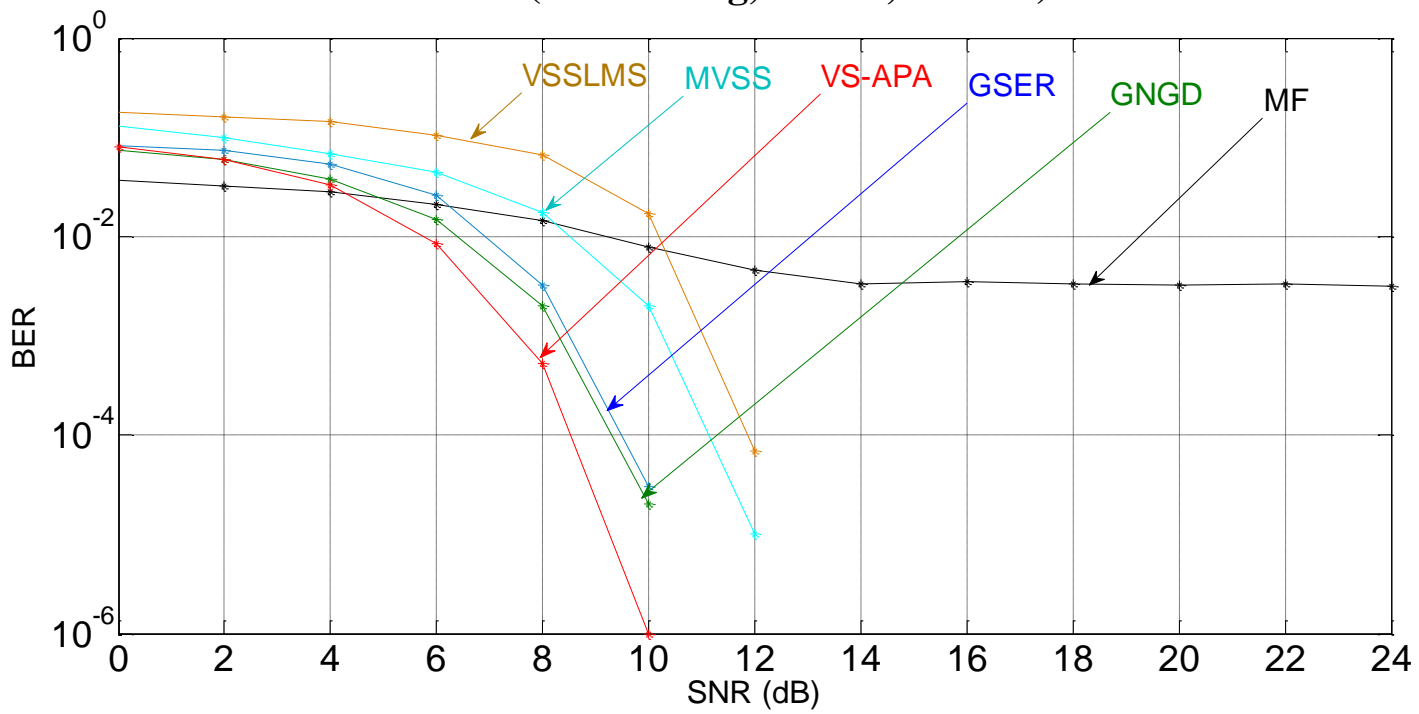

Fig. 8 Performance Comparison of the Various Adaptive Algorithms in CDMA Receivers (Flat Fading, 5 MAI, Gold31) 